\title{
Synthesis, Modeling and Anti-Tubulin Activity of a D-Seco Paclitaxel Analog
}

Luciano Barboni, ${ }^{\dagger *}$ Guido Giarlo ${ }^{\dagger}$ Massimo Ricciutelli, ${ }^{\dagger}$ Roberto Ballini, ${ }^{\dagger}$ Gunda I. Georg, ${ }^{\dagger *}$ David G. VanderVelde, ${ }^{\ddagger}$ Richard H. Himes, ${ }^{\S}$ Minmin Wang, ${ }^{\infty}$ Ami Lakdawala, ${ }^{\infty}$ and James P. Snyder ${ }^{\infty *}$

Dipartimento di Scienze Chimiche, Università di Camerino, 62032 Camerino (MC), Italy, Department of Medicinal Chemistry, University of Kansas, Drug Discovery Program, Higuchi Biosciences Center, University of Kansas, and Department of Molecular Biosciences, University of Kansas, Lawrence, Kansas 66045, and Department of Chemistry, Emory University, Atlanta, Georgia, 30322

$\begin{array}{ll}\text { General Methods } & \text { S1 } \\ \text { Preparation of Compound 5 } & \text { S1 } \\ \text { Preparation of Compound 6 } & \text { S2 } \\ \text { Preparation of Compound 7 } & \text { S2 } \\ \text { Preparation of Compound 8 } & \text { S2 } \\ \text { Preparation of Compound 1 } & \text { S3 }\end{array}$

\section{Experimental:}

General Methods. NMR spectra were taken on Bruker Avance 400 or Varian VXR 300 spectrometers. Cytotoxicity and tubulin binding were carried out according to established protocols. ${ }^{1}$ Commercially available reagents and solvents were used without further purification. All anhydrous reactions were performed under a positive pressure of nitrogen. $\mathrm{CH}_{2} \mathrm{Cl}_{2}$ was dried by distillation from $\mathrm{CaH}_{2}$, and THF by distillation from Nabenzophenone. Solvent evaporation was done under reduced pressure. Column chromatography was carried out with silica gel as the stationary phase.

Preparation of compound 5: To a stirred solution of compound 4 (655 $\mathrm{mg}, 0.611 \mathrm{mmol})$ in anhydrous $\mathrm{CH}_{2} \mathrm{Cl}_{2}$ at ambient temperature, $\mathrm{Bu}_{4}^{\mathrm{t}} \mathrm{NI}(1.830 \mathrm{mmol}, 675 \mathrm{mg})$ and $\mathrm{MgBr}_{2} \cdot \mathrm{Et}_{2} \mathrm{O}(0.775 \mathrm{mmol}, 200 \mathrm{mg})$ were added. The reaction mixture was stirred under $\mathrm{N}_{2}$ at $\mathrm{rt}$ for $5 \mathrm{~h}$ (monitored by TLC, hexane/EtOAc 4:1) then further $\mathrm{Bu}_{4}^{\mathrm{t}} \mathrm{NI}(0.542 \mathrm{mmol}$, $200 \mathrm{mg})$ and $\mathrm{MgBr}_{2} \cdot \mathrm{Et}_{2} \mathrm{O}(0.388 \mathrm{mmol}, 100 \mathrm{mg})$ were added. After $3 \mathrm{~h}$ the reaction mixture was diluted with $\mathrm{CH}_{2} \mathrm{Cl}_{2}$ and washed successively with water, $5 \% \mathrm{NaHCO}_{3}$ and brine. The organic phase was dried over anhydrous $\mathrm{Na}_{2} \mathrm{SO}_{4}$ and evaporated to dryness. The crude product was purified by silica gel chromatography (hexane/EtOAc 85:15) to give compound $5(638 \mathrm{mg}, 87 \%)$ as an amorphous solid. ${ }^{1} \mathrm{H} \mathrm{NMR}\left(\mathrm{CDCl}_{3}, 400 \mathrm{MHz}\right): \delta$ 0.33 (s, 3H), -0.13 (s, 3H), 0.59 (q, $J=7.9 \mathrm{~Hz}, 6 \mathrm{H}), 0.80$ (s, 9H), 0.94 (t, $J=7.9 \mathrm{~Hz}, 9 \mathrm{H})$, $1.13(\mathrm{~s}, 3 \mathrm{H}), 1.22(\mathrm{~s}, 3 \mathrm{H}), 1.37(\mathrm{~s}, 3 \mathrm{H}), 2.11(\mathrm{~m}, 1 \mathrm{H}), 2.19(\mathrm{~s}, 3 \mathrm{H}), 2.33(\mathrm{~m}, 1 \mathrm{H}), 2.48$ $(\mathrm{dd}, J=15.7,9.9 \mathrm{~Hz}, 1 \mathrm{H}), 2.50(\mathrm{bs}, 3 \mathrm{H}), 3.01(\mathrm{dd}, J=15.7,5.6 \mathrm{~Hz}, 1 \mathrm{H}), 3.85(\mathrm{~d}, J=10.8$ $\mathrm{Hz}, 1 \mathrm{H}), 3.98(\mathrm{~d}, J=5.0 \mathrm{~Hz}, 1 \mathrm{H}), 4.18(\mathrm{~d}, J=10.8 \mathrm{~Hz}, 1 \mathrm{H}), 4.29$ (s, 1H), 4.34 (d, $J=5.0$ $\mathrm{Hz}, 1 \mathrm{H}), 4.57$ (dd, $J=10.5,3.8 \mathrm{~Hz}, 1 \mathrm{H}), 4.61(\mathrm{~d}, J=2.0 \mathrm{~Hz}, 1 \mathrm{H}), 5.15$ (bs, 1H), 6.03 (m, $1 \mathrm{H}), 6.05(\mathrm{bd}, J=8.6 \mathrm{~Hz}, 1 \mathrm{H}), 6.60(\mathrm{~s}, 1 \mathrm{H}), 7.14(\mathrm{~d}, J=8.5 \mathrm{~Hz}, 1 \mathrm{H}), 7.26(\mathrm{~m}, 2 \mathrm{H}), 7.34$ $(\mathrm{bt}, J=7.4 \mathrm{~Hz}, 2 \mathrm{H}), 7.47(\mathrm{~m}, 3 \mathrm{H}), 7.52(\mathrm{~m}, 1 \mathrm{H}), 7.80(\mathrm{~m}, 2 \mathrm{H}) ;{ }^{13} \mathrm{C}$ NMR $(100 \mathrm{MHz}$, $\left.\mathrm{CDCl}_{3}\right): \delta-5.95,-5.58,5.16,6.76,13.77,14.38,17.82,18.22,19.77,20.77,25.53,26.37$, $32.81,39.05,41.07,43.88,45.38,55.62,62.15,71.02$ (2C), 71.30, 75.17, 75.24, 82.08, 
$89.89,127.06,127.22,127.58,128.31,128.77,131.86,133.16,133.81,138.84,143.38$, 151.97, 167.15, 169.02, 171.18, 200.76; MS (FAB+) m/z $1200.3(\mathrm{M}+\mathrm{H})^{+} ;[\alpha]^{20}{ }_{\mathrm{D}}+17.8$ (EtOAc, c 2.06).

Preparation of compound 6: To a solution of compound $5(617 \mathrm{mg}, 0.515 \mathrm{mmol})$ in EtOAc ( $30 \mathrm{ml}$, HPLC grade) at rt, $\mathrm{PtO}_{2}$ (Adams catalyst, $300 \mathrm{mg}$ ) was added. The suspension was stirred under $\mathrm{H}_{2}$ atmosphere (balloon pressure) for $3 \mathrm{~h}$; then additional $\mathrm{PtO}_{2}(100 \mathrm{mg}$ ) was added. After $17 \mathrm{~h}$ about $50 \%$ of starting material was present (TLC monitoring: hexane/EtOAc 4:1) and, since the catalyst was black and flaky, a further 150 $\mathrm{mg}$ of catalyst was added. After $24 \mathrm{~h}$ the mixture was filtered, evaporated to dryness and chromatographed (silica gel; hexane/EtOAc 85:15) to give starting material (137 mg,

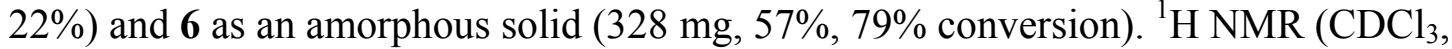
$300 \mathrm{MHz}): \delta-0.34(\mathrm{~s}, 3 \mathrm{H}),-0.15(\mathrm{~s}, 3 \mathrm{H}), 0.58(\mathrm{q}, J=7.9 \mathrm{~Hz}, 6 \mathrm{H}), 0.77$ (s, 9H), 0.93 (t, $J$ $=7.9 \mathrm{~Hz}, 9 \mathrm{H}), 1.11(\mathrm{~s}, 3 \mathrm{H}), 1.17(\mathrm{~s}, 3 \mathrm{H}), 1.36(\mathrm{~s}, 3 \mathrm{H}), 1.73(\mathrm{~s}, 3 \mathrm{H}), 2.16(\mathrm{~s}, 3 \mathrm{H}), 2.19(\mathrm{~m}$, 1H), 2.34 (m, 2H), 2.45 (bs, 3H), 2.94 (dd, $J=15.9,5.5 \mathrm{~Hz}, 1 \mathrm{H}), 3.57$ (d, $J=5.5 \mathrm{~Hz}$, $1 \mathrm{H}), 4.06(\mathrm{bs}, 1 \mathrm{H}), 4.40(\mathrm{~d}, J=5.5,1 \mathrm{H}), 4.56(\mathrm{dd}, J=10.4,3.9,1 \mathrm{H}), 4.62(\mathrm{~d}, J=1.8$, $1 \mathrm{H}), 4.63(\mathrm{~m}, 1 \mathrm{H}), 6.00(\mathrm{dd}, J=8.8,1.8 \mathrm{~Hz}, 1 \mathrm{H}), 6.08(\mathrm{~m}, 1 \mathrm{H}), 6.57(\mathrm{~s}, 1 \mathrm{H}), 7.13(\mathrm{~d}, J=$ $8.8 \mathrm{~Hz}, 1 \mathrm{H}), 7.24(\mathrm{~m}, 2 \mathrm{H}), 7.31(\mathrm{~m}, 2 \mathrm{H}), 7.46(\mathrm{~m}, 2 \mathrm{H}), 7.53(\mathrm{~m}, 2 \mathrm{H}), 7.77(\mathrm{~m}, 2 \mathrm{H}) ;{ }^{13} \mathrm{C}$ NMR $\left(100 \mathrm{MHz} \mathrm{CDCl}_{3}\right): \delta-6.02,-5.69,5.10,6.67,12.61,17.61,18.12,19.53,20.72$, $25.43,25.58,26.39,36.46,39.15,40.73,46.07,47.46,55.03,62.08,70.54,71.07,71.58$, 75.24, 75.28, 82.38, 90.10, 126.99, 127.23, 127.45, 128.17, 128.71, 131.77, 132.94, 133.80, 138.78, 142.97, 152.34, 166.56, 168.99, 171.30, 201.46; MS (FAB+) m/z 1074.3 $(\mathrm{M}+\mathrm{H})^{+} ;[\alpha]^{20}{ }_{\mathrm{D}}+9.1\left(\mathrm{CHCl}_{3}, c 1.13\right)$.

Preparation of compound 7: To a stirred solution of compound 6 (328 mg, $0.306 \mathrm{mmol})$ in DMPU (20 ml) under $\mathrm{N}_{2}$ atmosphere, $\mathrm{NaBH}_{3} \mathrm{CN}(77 \mathrm{mg}, 1.223 \mathrm{mmol})$ was added. The reaction mixture was stirred overnight at $70{ }^{\circ} \mathrm{C}$ under $\mathrm{N}_{2}$ atmosphere, then the reaction mixture was quenched with $2 \mathrm{~N} \mathrm{HCl}$ and extracted with EtOAc. The organic solution was washed consecutively with $5 \% \mathrm{NaHCO}_{3}, \mathrm{H}_{2} \mathrm{O}$ and brine, dried over $\mathrm{Na}_{2} \mathrm{SO}_{4}$ and evaporated to dryness. The crude product was purified by silica gel chromatography (hexane/EtOAc 85:15) to yield compound $7\left(210 \mathrm{mg}, 73 \%\right.$ ) as an amorphous solid. ${ }^{1} \mathrm{H}$ NMR $\left(\mathrm{CDCl}_{3}, 400 \mathrm{MHz}\right): \delta-0.29(\mathrm{~s}, 3 \mathrm{H}),-0.11(\mathrm{~s}, 3 \mathrm{H}), 0.64(\mathrm{q}, J=8.0 \mathrm{~Hz}, 6 \mathrm{H}), 0.84$ (s, $9 \mathrm{H}), 0.95(\mathrm{t}, J=8.0 \mathrm{~Hz}, 9 \mathrm{H}), 1.18(\mathrm{~s}, 3 \mathrm{H}), 1.22(\mathrm{~s}, 3 \mathrm{H}), 1.25(\mathrm{~s}, 3 \mathrm{H}), 1.64(\mathrm{~s}, 3 \mathrm{H}), 2.14$ (bs, 3H), $2.20(\mathrm{~s}, 3 \mathrm{H}), 2.52(\mathrm{dd}, J=15.8,10.0 \mathrm{~Hz}, 1 \mathrm{H}), 2.94$ (dd, $J=15.8,4.2 \mathrm{~Hz}, 1 \mathrm{H})$, $2.98(\mathrm{~d}, J=6.2 \mathrm{~Hz}, 1 \mathrm{H}), 3.84(\mathrm{~s}, 1 \mathrm{H}), 4.31(\mathrm{~d}, J=6.2 \mathrm{~Hz}, 1 \mathrm{H}), 4.45(\mathrm{~d}, J=1.5 \mathrm{~Hz}, 1 \mathrm{H})$, 4.78 (bs, 1H), 5.52 (dd, $J=10.3,1.5 \mathrm{~Hz}, 1 \mathrm{H}), 5.69$ (dd, $J=10.3,1.9 \mathrm{~Hz}, 1 \mathrm{H}), 5.89$ (bd, $J$ $=10.0 \mathrm{~Hz}, 1 \mathrm{H}), 5.99(\mathrm{bd}, J=8.5 \mathrm{~Hz}, 1 \mathrm{H}), 6.58(\mathrm{~s}, 1 \mathrm{H}), 7.26(\mathrm{~m}, 2 \mathrm{H}), 7.35(\mathrm{~m}, 4 \mathrm{H}), 7.47$ $(\mathrm{m}, 2 \mathrm{H}), 7.53(\mathrm{~m}, 1 \mathrm{H}), 7.78(\mathrm{~m}, 2 \mathrm{H}) ;{ }^{13} \mathrm{C} \mathrm{NMR}\left(100 \mathrm{MHz}, \mathrm{CDCl}_{3}\right): \delta-5.99,-5.69,5.19$, $6.66,12.75,16.77,18.14,18.90,20.79,25.44,27.06,27.25,32.70,40.55,51.06,54.95$, $61.51,70.97,71.81,72.15,75.34,75.69,81.80,89.84,126.56,127.00,127.62,128.33$, $125.66,128.70,131.80,132.43,133.82,138.18,139.63,140.60,152.71,166.93,169.20$, 170.41, 203.16; MS (FAB+) m/z $946.7(\mathrm{M}+\mathrm{H})^{+} ;[\alpha]_{\mathrm{D}}^{20}-19.5\left(\mathrm{CHCl}_{3}, c 0.57\right)$.

Preparation of compound 8: To a stirred solution of compound 7 (263 mg, $0.278 \mathrm{mmol}$ ) in toluene $(30 \mathrm{ml})$ under $\mathrm{N}_{2}$ atmosphere, DMAP $(1360 \mathrm{mg}, 11.130 \mathrm{mmol})$ and acetic anhydride $\left(\mathrm{Ac}_{2} \mathrm{O}\right)(1136 \mathrm{mg}, 11.130 \mathrm{mmol}, 1.05 \mathrm{ml})$ were added. The reaction mixture was stirred overnight at $80^{\circ} \mathrm{C}$ under $\mathrm{N}_{2}$ atmosphere and further $\mathrm{Ac}_{2} \mathrm{O}(1 \mathrm{ml})$ and DMAP (1350 mg) were added. After $24 \mathrm{~h}$ further $\mathrm{Ac}_{2} \mathrm{O}(0.5 \mathrm{ml})$ and DMAP $(700 \mathrm{mg})$ were added. After $5 \mathrm{~h}$ the reaction mixture was concentrated under vacuum, diluted with 
EtOAc and extracted consecutively with $2 \mathrm{~N} \mathrm{HCl}, 5 \% \mathrm{NaHCO}_{3}, \mathrm{H}_{2} \mathrm{O}$ and brine, dried over $\mathrm{Na}_{2} \mathrm{SO}_{4}$ and evaporated to dryness. The crude product was purified by silica gel chromatography (hexane/EtOAc 85:15) to give starting material $(80 \mathrm{mg})$ and compound $8(80 \mathrm{mg}, 30 \%)$ as an amorphous solid. ${ }^{1} \mathrm{H}$ NMR $\left(\mathrm{CDCl}_{3}, 300 \mathrm{MHz}\right): \delta-0.26(\mathrm{~s}, 3 \mathrm{H}),-$ $0.05(\mathrm{~s}, 3 \mathrm{H}), 0.58(\mathrm{q}, J=7.9 \mathrm{~Hz}, 6 \mathrm{H}), 0.80(\mathrm{~s}, 9 \mathrm{H}), 0.88(\mathrm{t}, J=7.9 \mathrm{~Hz}, 9 \mathrm{H}), 1.22(\mathrm{~s}, 3 \mathrm{H})$, $1.24(\mathrm{~s}, 3 \mathrm{H}), 1.27(\mathrm{~s}, 3 \mathrm{H}), 1.83(\mathrm{~s}, 3 \mathrm{H}), 1.98(\mathrm{bs}, 3 \mathrm{H}), 2.15(\mathrm{~s}, 3 \mathrm{H}), 2.16(\mathrm{~s}, 3 \mathrm{H}), 2.58(\mathrm{dd}$, $J=15.6,9.8 \mathrm{~Hz}, 1 \mathrm{H}), 2.98(\mathrm{dd}, J=15.6,6.7 \mathrm{~Hz}, 1 \mathrm{H}), 3.01(\mathrm{~d}, J=6.7 \mathrm{~Hz}, 1 \mathrm{H}), 4.36(\mathrm{~d}, J$ $=6.7 \mathrm{~Hz}, 1 \mathrm{H}), 4.62(\mathrm{~d}, J=1.8 \mathrm{~Hz}, 1 \mathrm{H}), 4.63(\mathrm{bs}, 1 \mathrm{H}), 5.57(\mathrm{~d}, J=9.1 \mathrm{~Hz}, 1 \mathrm{H}), 5.67(\mathrm{dd}$, $J=9.1,1.2 \mathrm{~Hz}, 1 \mathrm{H}), 6.13$ (bt, $J=8.5 \mathrm{~Hz}, 1 \mathrm{H}), 6.40$ (dd, $J=9.2,1.8 \mathrm{~Hz}, 1 \mathrm{H}), 6.47$ (s, $1 \mathrm{H}), 7.10(\mathrm{~d}, J=9.2,1 \mathrm{H}), 7.31(\mathrm{~m}, 5 \mathrm{H}), 7.49(\mathrm{~m}, 3 \mathrm{H}), 7.79(\mathrm{~m}, 2 \mathrm{H}) ;{ }^{13} \mathrm{C} \mathrm{NMR}(75 \mathrm{MHz}$, $\left.\mathrm{CDCl}_{3}\right): \delta-5.57,-5.11,5.26,6.68,13.46,15.96,18.09,20.05,20.79,23.12,25.57,25.65$, 26.17, 32.29, 41.17, 48.89, 55.54, 61.23, 70.51, 72.08, 75.16, 75.43, 81.25, 83.30, 89.86, $126.45,127.01,127.94,128.64,128.78,129.13,130.78,131.83,132.23,134.02,138.07$, 142.24, 152.66, 166.65, 169.16, 169.33, 171.57, 202.51; MS (ES+) m/z 1010.7 (M + $\mathrm{Na})^{+} ;[\alpha]^{20}{ }_{D}-33.6\left(\mathrm{CHCl}_{3}, c 1.12\right)$.

Preparation of compound 1: To a stirred solution of compound 8 (80 mg, $0.081 \mathrm{mmol})$ in anhydrous THF $(3 \mathrm{ml})$ at $-78{ }^{\circ} \mathrm{C}$ under $\mathrm{N}_{2}$ atmosphere, phenyllitium ( $\mathrm{PhLi}, 0.405$ mmol, $225 \mu \mathrm{l}$ of $1.8 \mathrm{M}$ solution in cyclohexane-ether) was added. After stirring at $-78^{\circ} \mathrm{C}$ for $1 \mathrm{~h}$, TLC (hexane/EtOAc 4:1) showed the initial formation of a compound with a lower rf that that of the starting material and further $100 \mu \mathrm{l}$ of $\mathrm{PhLi}$ were added. After 1.5 h $100 \mu \mathrm{l}$ of $\mathrm{PhLi}$ were added and the reaction mixture was stirred at $-78{ }^{\circ} \mathrm{C}$ for further 30 minutes (the TLC showed the formation of many spots). The mixture was then quenched with EtOAc and the organic phase was washed with $5 \% \mathrm{NaHCO}_{3}$, water and brine. The EtOAc layer was then dried over $\mathrm{Na}_{2} \mathrm{SO}_{4}$ and evaporated to dryness. The mixture was dissolved in anhydrous THF $(1 \mathrm{ml})$ in a teflon vial, the solution was stirred at $0{ }^{\circ} \mathrm{C}$ and a cold solution $\left(-20^{\circ} \mathrm{C}\right)$ of hydrogen fluoride-pyridine $(\mathrm{HF} / \mathrm{Py}, 100 \mu \mathrm{l})$ was added. The reaction mixture was stirred at room temperature for $3 \mathrm{~h}$ and then further $50 \mu \mathrm{lof} \mathrm{HF} / \mathrm{Py}$ were added. After $1 \mathrm{~h}$, the mixture was diluted with EtOAc $(5 \mathrm{ml})$ and neutralised with a saturated $\mathrm{NaHCO}_{3}$ solution. The solution was then extracted successively with $2 \mathrm{~N} \mathrm{HCl}$, $5 \% \mathrm{NaHCO}_{3}$, water and brine. The EtOAC layer was then dried over $\mathrm{Na}_{2} \mathrm{SO}_{4}$ and evaporated to dryness. The crude product was purified by preparative TLC (silica gel, hexane/EtOAc 2:3) to give compound 1 (2 mg, 3\%). ${ }^{1} \mathrm{H}$ NMR $\left(\mathrm{CDCl}_{3}, 300 \mathrm{MHz}\right): \delta 1.13$ (s, 3H, H-16), 1.15 (s, 3H, H-17), 1.34 (s, 3H, H-19), 1.44 (s, 3H, H-20), 1.65 (bs, 3H, H18), 2.21 (s, 3H, 4-OAc), 2.28 (dd, $J=15.5,7.3 \mathrm{~Hz}, 1 \mathrm{H}, \mathrm{H}-14), 2.38$ (dd, $J=15.5,9.4$ $\mathrm{Hz}, 1 \mathrm{H}, \mathrm{H}-14), 3.91$ (d, $J=7.4 \mathrm{~Hz}, 1 \mathrm{H}, \mathrm{H}-3), 4.02$ (d, $J=1.8 \mathrm{~Hz}, 1 \mathrm{H}, 10-\mathrm{OH}), 4.41$ (m, $1 \mathrm{H}, \mathrm{H}-7), 4.43$ (d, $\left.J=3.2 \mathrm{~Hz}, 1 \mathrm{H}, 2^{\prime}-\mathrm{OH}\right), 4.77$ (dd, $\left.J=3.2,2.4 \mathrm{~Hz}, 1 \mathrm{H}, \mathrm{H}-2^{\prime}\right), 5.31$ (d, $J$ $=1.8 \mathrm{~Hz}, 1 \mathrm{H}, \mathrm{H}-10), 5.53(\mathrm{dd}, J=10.4,1.2 \mathrm{~Hz}, 1 \mathrm{H}, \mathrm{H}-6), 5.55$ (d, $J=7.4 \mathrm{~Hz}, 1 \mathrm{H}, \mathrm{H}-2)$, $5.70(\mathrm{dd}, J=10.4,2.4 \mathrm{~Hz}, 1 \mathrm{H}, \mathrm{H}-5), 5.83$ (dd, $J=9.2,2.4 \mathrm{~Hz}, 1 \mathrm{H}, \mathrm{H}-3$ '), 6.07 (bt, $J=8.2$ $\mathrm{Hz}, 1 \mathrm{H}, \mathrm{H}-13), 7.25-7.62(\mathrm{~m}, 12 \mathrm{H}, \mathrm{NH}+11$ aromatic protons), $7.82(\mathrm{bd}, J=7.9 \mathrm{~Hz}, 2 \mathrm{H}$, $o-\mathrm{OBz}), 8.07(\mathrm{bd}, J=7.7 \mathrm{~Hz}, 2 \mathrm{H}, o-\mathrm{NHBz}) .{ }^{13} \mathrm{C} \mathrm{NMR}\left(75 \mathrm{MHz}, \mathrm{CDCl}_{3}\right): \delta 12.46(\mathrm{q}, \mathrm{C}-$ 19), 15.00 (q, C-18), 19.16 (q, C-16), 22.88 (q, 4-OAc), 26.70 (q, C-17), 28.34 (q, C-20), 36.19 (t, C-14), 42.52 (s, C-15), 49.30 (d, C-3), 54.61 (d, C-3'), 60.02 (s, C-8), 71.46 (d, C-13), 72.80 (d, C-7), 73.99 (d, C-2'), 74.34 (d, C-10), 74.81 (d, C-2), 78.83 (s, C-1), 83.32 (s, C-4), 126.04 (d, C-6), 127.02 (d, $m-\mathrm{NBz}), 128.05$ (d, $p-\mathrm{Ph}), 127.21,128.62$, 128.68 (2 signals), 129.65, 129.77 (o-OBz, o-NHBz, o-Ph, $m$-Ph, C1-OBz, C1-Ph), 130.02 (d, $m$-OBz), 131.85 (d, p-NBz), 133.56 (d, p-OBz), 133.95 (d, C-5), 134.82 (s, C- 
11), 137.14 (s, C-12), 138.27 (s, C1-NHBz), 166.40 (s, NHBz), 167.34 (s, OBz), 170.74 (s, 4-OAc), 171.24 (s, C-1'), $210.73(\mathrm{~s}, \mathrm{C}-9)$; $[\alpha]^{20}{ }_{\mathrm{D}}-55.8\left(\mathrm{CHCl}_{3}, c 0.09\right)$. High resolution mass spectrum: $\mathrm{ES}+$ in methanol, $5 \mathrm{mM} \mathrm{NH}_{4} \mathrm{OAc}$ : 813.3612, calc. for $\mathrm{M}+\mathrm{NH}_{4}, \mathrm{C}_{45} \mathrm{H}_{53} \mathrm{~N}_{2} \mathrm{O}_{2}=813.3599(1.3 \mathrm{mmu})$

1 Liu, Y.; Ali, S. Y.; Boge, T. C.; Georg, G. I.; Victory, S.; Zygmunt, J.; Marquez, R. T.; Himes, R. H. A Systematic SAR Study of C10 Modified Paclitaxel Analogues Using a Combinatorial Approach. Combi. Chem. \& High Thr. Scr. 2002, 5, 39-48. 